This item was submitted to Loughborough's Research Repository by the author.

Items in Figshare are protected by copyright, with all rights reserved, unless otherwise indicated.

\title{
A mathematical model for the human menstrual cycle
}

\section{PLEASE CITE THE PUBLISHED VERSION}

http://dx.doi.org/10.1093/imammb/dqs048

\section{PUBLISHER}

(C) The Authors. Published by Oxford University Press on behalf of the Institute of Mathematics and its Applications

\section{VERSION}

AM (Accepted Manuscript)

\section{PUBLISHER STATEMENT}

This work is made available according to the conditions of the Creative Commons Attribution-NonCommercialNoDerivatives 4.0 International (CC BY-NC-ND 4.0) licence. Full details of this licence are available at: https://creativecommons.org/licenses/by-nc-nd/4.0/

\section{LICENCE}

CC BY-NC-ND 4.0

\section{REPOSITORY RECORD}

Chen, C.Y., and John P. Ward. 2019. "A Mathematical Model for the Human Menstrual Cycle". figshare. https://hdl.handle.net/2134/16305. 


\title{
A mathematical model for the human menstrual cycle
}

\author{
C.Y. Chen* and J.P. Ward ${ }^{+}$ \\ * Department of Applied Mathematics, The National University of Kaohsiung, \\ Kaohsiung, Taiwan \\ + Department of Mathematical Sciences, Loughborough University, \\ Loughborough, U.K.
}

January 18, 2013

\begin{abstract}
A simple mathematical model framework is developed to describe the hormonal interactions of the human menstrual cycle along the hypothalamus-pituitary-ovaries axis. The framework is designed so that it can be readily extended to model processes that disrupt the normal functioning cycle. The model in its most basic formulation exhibits multiple periodic solutions, one of which shows the key characteristics of a menstrual cycle, whilst the others indicate possible abnormalities sometimes observed in women of reproductive age. The basic model is extended to encompass receptor down-regulation as a mechanism to describe the desensitisation of the pituitary to continuous stimulation of hypothalamic hormone, a hormonal therapy that is commonly prescribed prior to the surgical procedure for the removal of uterine myomas. Though the mechanisms for desensitisation are likely to be more complex, the model results are in good qualitative agreement with physiological observations.
\end{abstract}

Keywords: mathematical modelling, delay differential equations, Hopf bifurcation, periodic solutions, menstrual cycle

\section{Introduction}

The female menstrual cycle, regulated by the endocrine system, is maintained through a sequence of complex interactions of hormones. The predominant hormones involved in these events taking place along the hypothalamus-pituitary-ovaries axis of the endocrine system are gonadotropin releasing hormone $(\mathrm{GnRH})$, follicle stimulating hormone (FSH), luteinizing hormone (LH) and ovarian hormones such as estrogen(E2), progesterone (P4) and inhibin. GnRH is believed to be responsible for the synthesis and release of FSH and LH in the anterior pituitary. The released FSH stimulates the recruitment and growth of follicles in the ovary while LH promotes the ovulation and the subsequent conversion of follicles into corpus lutea. Follicles and corpus lutea secrete ovarian hormones which, in turn, feedback on both GnRH neurons and the pituitary, directly and indirectly, fine-tuning the response of GnRH, LH and FSH, maintaining the cyclic nature of the reproductive system $[23,26]$. The regulating process is highly interactive and complex. Our aim here is to formulate a simple, minimal model that encapsulates the key underlying dynamics of the menstrual cycle, whilst describing explicitly the role of the principal hormones governing the process, the feedback of estrogen and progesterone on FSH and LH and, in particular, the regulatory effect of $\mathrm{GnRH}$ on the responsiveness of the pituitary. We 
first discuss in more detail the relevant background of the menstrual cycle and follow this with a brief overview of modelling literature.

$\mathrm{GnRH}$ is a hypothalamic hormone released by neurons in a pulsatile manner with varying frequencies and amplitudes. The $\mathrm{GnRH}$ neuron activities are regulated by the afferent neuronal systems which, as many studies have demonstrated, are sensitive to the feedback from the ovarian hormones such as estrogen and progesterone $[16,19,31]$. While estrogen is observed to promote the frequency of $\mathrm{GnRH}$ release from the neurons, progesterone is reported to have a negative effect in reducing GnRH frequency. The varying frequencies, accompanied by a preovulatory surge, subsequently result in the differential release of FSH and LH in the pituitary; at lower frequency, FSH secretion is favoured while at higher frequency, LH secretion increases disproportionately.

The exact mechanism that leads to the differential release of FSH and LH in the pituitary is still very much under debate. GnRH initiates response at the anterior pituitary cells by binding to a family of membrane receptors called G-protein coupled receptors which stimulate the downstream responses by coupling to G-protein on the cell membrane. Multiple activation of G-protein subtypes by a single receptor and the subsequent cross-talk between their corresponding signalling pathways leading to multiple physiological functions have been observed in experiments [21, 27]. Feedback of ovarian hormones also play a part in controlling the pituitary response to $\mathrm{GnRH}$; in particular, both estrogen and inhibin have been reported to exert negative feedback on FSH release and secretion with estrogen playing the greater role in the control of FSH concentration levels [1, 26, 38]. The combined effects of these signalling mechanisms and feedback control of estrogen and progesterone are likely to be instrumental in orchestrating the differential control of FSH and LH in the pituitary[13].

The menstrual cycle is commonly divided into two phases: the follicular phase and the luteal phase separated by a period of ovulation with the follicular phase characterised by the growth and development of follicles while the luteal phase by the formation of corpus lutea until luteolysis (or pregnancy). FSH and LH work in concert to control the development of ovarian follicles and corpus lutea. Stimulated by FSH, the newly recruited follicles grow to maturation while secreting estrogen. As ovulation approaches, one of these follicles becomes dominant and grows more rapidly than the others, until, stimulated by the surge of LH, it ruptures and releases the egg contained within it. The ruptured follicle is then transformed into the corpus luteum which secretes estrogen and progesterone that regulate hypothalamus neuron activities and suppress the pituitary secretion.

Understanding the cascade of events has helped to design therapies for conditions that are hormone-related. For example, contraceptive pills (of progesterone) are formulated to disrupt the reproductive cycle, using the negative feedback of progesterone on $\mathrm{GnRH}$ to suppress the hormone levels [8]. It is also a common practice in the treatment of uterine myoma to administer exogenous GnRH analog before surgery to reduce myoma size and minimise surgical risk [15].

Mathematical models have been developed to address different issues that arise in the study of the reproductive system. Khadra and $\operatorname{Li}[18,22]$ based on physiological data from experiments, developed models focusing on the pulsatile secretion of $\mathrm{GnRH}$ from hypothalamic neurons. A different approach was taken by Clement and Francoise [6] who formulated a phenomenological model to regulate the frequency and amplitude of GnRH by coupling two systems of FitzHughNagumo models. A model proposed by Bertram and Li [3] was used to investigate the effect of activin and follistatin on the differential responses of LH and FSH in the pituitary. Models focused on periodic solutions of the entire menstrual cycle have also been developed. Clark et al. [4], for example, put forward a nonlinear system of 13 delay differential equations to describe 
the concentration of hormones and other components that are involved in the maintenance of the menstrual cycle without explicitly considering the role of GnRH. This model is further extended by Reinecke and Deuflhard [29] to include a stochastic process for the generation of GnRH. The models described in $[4,29]$ are very complex, involving a number of variables accounting for the main hormones, which are mostly measurable, and a large number of proposed follicular states, which are not so readily discernible from data or observation; nevertheless, with parameter fitting, good quantitative agreement with data are predicted by these models. However, we viewed these models as being rather too complex to be a desirable starting point to investigate additional processes, such as GnRH desensitisation discussed in this paper.

The aim of the current study is to develop a relatively simple mathematical model, incorporating the key biological processes, that exhibits stable periodic solutions that are characteristic of the menstrual cycle and, in particular, can be used as a basic framework to investigate processes that perturb the cycle. The basic model will be extended to investigate the pituitary desensitisation to GnRH stimulation, which to our knowledge has not been included in the modelling of the menstrual cycle, enabling us to simulate GnRH agonist treatment. This model will be incorporated into a on-going study on the growth and treatment of myomas in the uterus, for which the simulation of GnRH treatments and the periodic secretion of estrogen under normal physiological condition are required. The sections in this paper are arranged as follow. In Section 2, we outline the assumptions and present the basic model of the menstrual cycle consisting of three delay differential equations to describe the hormonal interactions of the menstrual cycle. Numerical simulations and a discussion of the first stage modelling are presented in Section 2.1 with a brief bifurcation analysis carried out in Section 2.2. The model is extended in Section 3 by encompassing a mechanism for GnRH treatment with numerical simulations of the treatment illustrated in Section 3.1. A summary and discussion is given in Section 4.

\section{Formulation of the basic model}

The primary aim of this study is to construct a mathematical model which is notably simpler than existing models, yet is able to describe the interactions of the key components and reproduces many of the observed and measured features of the menstrual cycle. Moreover, the assumptions are based on experimental observations while simple kinetics terms are used to describe qualitatively the known causes and effects within the system. We focus on the actions of five dominant hormones, namely GnRH, FSH, LH, estrogen (E2) and progesterone (P4), and describe the ovarian stages in terms of three variables representing masses of the follicle, follicular scar and corpus luteum. The main regulatory pathways between the hormones and ovarian stages are illustrated in Figure 1 and this will form the basis of the modelling to follow. There are several other components known to be involved in the regulation of the cycle, e.g. the enzymes activin, inhibin 1 and 2 and follistatin [37], but we assume these play a secondary role in the process and can be neglected in a first approximation. In brief, the modelling setup assumes that follicular maturation and corpus luteum developments are governed by the hypothalamus, via pulsatile GnRH release, which stimulates the pituitary for the production of FSH and LH. FSH promotes the maturation of the follicle and LH governs the rupturing of the follicle. In turn, the production of estrogen and progesterone by the follicle and corpus luteum promotes and inhibits, respectively, the release rate of $\mathrm{GnRH}$ from the hyperthalamus. We will not attempt to model the pulsatile behaviour of $\mathrm{GnRH}$, and the GnRH variable in the model can be viewed as a moving average concentration discussed below. Table 1 lists the variables to 


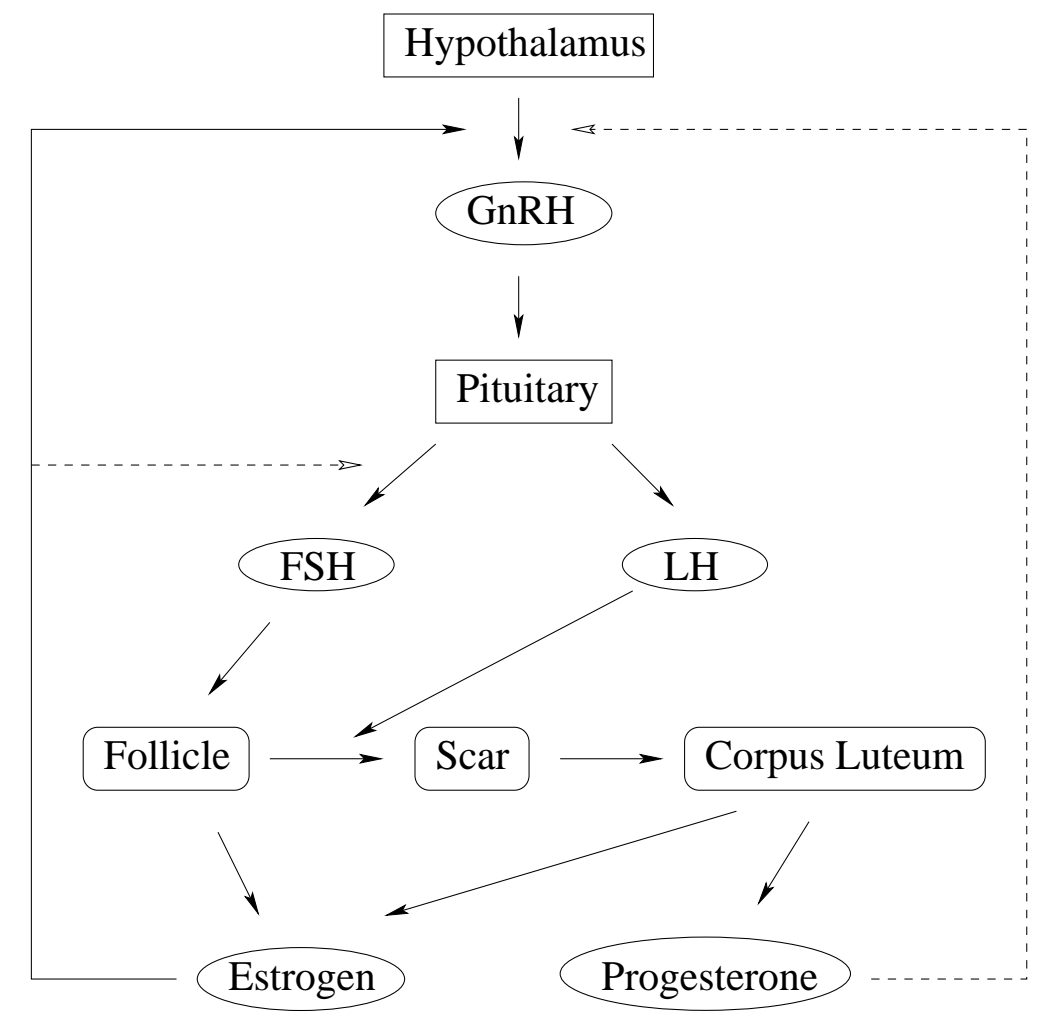

Figure 1: Simplified regulatory pathway diagram of the menstrual cycle used as a basis for the mathematical model. The secretion of $\mathrm{GnRH}$ from the hypothalamus stimulates the pituitary for the release of FSH and LH. FSH promotes the growth of follicle and its rupture, promoted by $\mathrm{LH}$, leads to the formation of scar and the subsequent conversion into corpus luteum. Estrogen and progesterone, secreted by the follicle and corpus luteum, feedback on hypothalamus and pituitary to regulate GnRH and FSH production. Negative feedbacks are indicated by broken lines, otherwise the effect is positive.

\begin{tabular}{|l|l|}
\hline$F$ & Dominant follicle mass \\
$R$ & Ruptured follicle or scar mass \\
$C$ & Corpus body/luteum mass \\
\hline$E$ & Estrogen $\left(\mathrm{E}_{2}\right)$ concentration \\
$P$ & Progesterone $\left(\mathrm{P}_{4}\right)$ concentration \\
$G$ & Mean GnRH concentration in the portal blood \\
$L$ & Luteinising Hormone (LH) concentration \\
$S$ & Follicle Stimulating Hormone (FSH) concentration \\
\hline
\end{tabular}

Table 1: Table of the variables. In the model, the top three variables change over the (slow) time-scale of interest and the five below are assumed to be quasi-steady.

be used in the model. The follicular and luteal development occurs over a timescale of weeks, whilst the hormonal responses are expected to occur in timescales of minutes or hours; i.e. the hormonal response will equilibrate rapidly to any changes to the follicle or corpus luteum. We thus treat the hormone levels as quasi-steady functions of follicular and luteal states. The processes of gene regulation and hormonal production form complex, feedback pathways working on 
multiple timescales, e.g. gene transcription (minutes) and enzymatic reactions (seconds). Over longer timescales, in our case $O$ (days), the effective dynamics are often well described using Hill functions, which will be adopted here. The kinetics used to describe promotion $\left(H^{+}\right)$and inhibition $\left(H^{-}\right)$are taken to be proportional to normalised Hill functions (i.e. $0 \leq H^{+/-} \leq 1$ ), defined as follows

$$
H^{+}\left(x ; x_{c}, m\right)=\frac{\left(x / x_{c}\right)^{m}}{1+\left(x / x_{c}\right)^{m}}, \quad H^{-}\left(x ; x_{c}, m\right)=\frac{1}{1+\left(x / x_{c}\right)^{m}},
$$

where both functions are strictly monotonic, with $H^{+}=0$ and $H^{-}=1$ at $x=0$ and $H^{+}=1$ and $H^{-}=0$ as $x \rightarrow \infty$. The constant $x_{c}$ is the Hill function coefficient, such that $H^{+/-}\left(x_{c} ; x_{c}, m\right)=$ $1 / 2$, and $m$ is the Hill function exponent that governs how rapidly the function jumps from 0 to 1 or vice versa in the vicinity of $x=x_{c}$. Small integer exponents $m$ (e.g. $m=1$ or $m=2$ ) can be relatively straightforward to explain using ideas from enzyme kinetics, whilst larger values would suggest that the underlying processes are much more complex, however, many complex processes are well described using small values of $m[20]$. Based on these assumptions and the pathway diagram shown in Figure 1, we assume the ovarian state variables to evolve according to

$$
\begin{aligned}
\frac{d F}{d t} & =\left(\beta_{f}+\beta_{f s} H^{+}\left(S ; S_{c}, m_{S}\right)\right) F-\gamma_{f r} H^{+}\left(L ; L_{c}, m_{L}\right) F^{2} \\
\frac{d R}{d t} & =\gamma_{f r} H^{+}\left(L ; L_{c}, m_{L}\right) F^{2}-\gamma_{r c} R \\
\frac{d C}{d t} & =\gamma_{r c} R-\delta_{c} C .
\end{aligned}
$$

The variables $F, R$ and $C$ represent masses of follicle, scar and luteal material, $S$ and $L$ in the Hill functions are the concentration levels of FSH and LH. In (2.1)-(2.3), the production, conversion and decay rate constants are represented by $\beta, \gamma$ and $\delta$, respectively, and the subscripts relate these rate constants to the relevant hormones or ovarian states; for example, $\beta_{f s}$ is the FSH $(S)$ enhanced follicle $(F)$ growth rate while $\delta_{c}$ is the corpus luteum $(C)$ decay rate. This subscript notation is used throughout the paper unless stated otherwise; the definitions of all the parameters are listed in Table 2 in the appendix. The development of the ovulatory follicle begins with the recruitment of a number of primordial follicles, which over the next 300-400 days grow through a number of phases, during which most of the recruited follicles will be lost (via atresia) [14]. About 10-15 days before ovulation (i.e. at the start of the follicular phase) one of the few remaining follicles will become dominant and begin to grow much more rapidly, subsequently producing most of the secreted estrogen during the follicular phase. The variable $F$ represents the mass of the portion of the dominant follicle that will ultimately develop to the corpus luteum; all activities by follicles, including the remainder of the dominant follicle and the non-dominant follicles, although not explicitly modelled, are assumed to occur at rates proportional to $F$. The first term on the right-hand side of Equation (2.1) describes the recruitment and growth of this follicular mass, combining basal and FSH-stimulated enhanced growth. The second term describes the rupturing of the follicle, i.e. conversion to scar, due to the action of LH reflecting egg release. We have assumed that this conversion rate from follicle to scar is proportional to $F^{2}$, as the predicted hormone profiles using this form was found to match well with data in the vicinity of ovulation. A biological interpretation of this form is that the follicular cells increase their sensitivity to LH as the follicle enlarges and matures, where the term used here suggests that the per capita level of follicular sensitivity is proportional to $F$; this reflects the observation 
that only larger follicles ovulate in response to LH [14]. Equations (2.2) and (2.3) describe the transfer of follicular material, via action of LH, to form scar tissue (mass $R$ ) and then the scar to corpus luteum (mass $C$ ); these processes are assumed to occur naturally without further intervention of hormones.

The kinetics in equations (2.1) and (2.2) involve FSH $(S)$ and LH $(L)$, that are released by the pituitary constitutively and in response to $\mathrm{GnRH}$ levels, denoted by $G$. The binding of GnRH with the surface receptors at the pituitary initiates a cascade of events during which the gonadotropin are synthesized first and stored in a reserve pool before being released into the blood stream. As our modelling here concentrates on the serum levels of FSH and LH, denoted by $S$ and $L$ respectively, it seems reasonable to model this using a time lag between $\mathrm{GnRH}$ stimulation and hormone production in the blood stream; in addition, the release of FSH is also inhibited by the current concentration of estrogen, denoted by $E[13,38]$. Note that both the differential stimulation of GnRH on the pituitary hormones and the feedback of estrogen involve further downstream interactions that are not explicitly modelled here $[13,16]$, instead, the effect will be represented using Hill functions. Taking FSH and LH at quasi-steady levels, we write

$$
\begin{aligned}
& S=\left(S_{\text {min }}+\left(S_{\text {max }}-S_{\text {min }}\right) H^{+}\left(G\left(t-\tau_{F S H}\right) ; G_{c S}, m_{G S}\right)\right) H^{-}\left(E ; E_{c S}, m_{E S}\right), \\
& L=L_{\text {min }}+\left(L_{\text {max }}-L_{\text {min }}\right) H^{+}\left(G\left(t-\tau_{L H}\right) ; G_{c L}, m_{G L}\right),
\end{aligned}
$$

where $\tau_{F S H}$ and $\tau_{L H}$ are the lag time between pituitary excitation by GnRH and the release of FSH and LH, respectively; $S_{\min }$ and $S_{\max }$ are the maximum and minimum FSH levels and $L_{\min }$ and $L_{\max }$ are those of LH. These formulations ensure that $S \in\left(S_{\min }, S_{\max }\right)$ and $L \in$ $\left(L_{\min }, L_{\max }\right)$, where the minimum values reflect background levels in the absence of $\mathrm{GnRH}$; in humans, measured data indicate that these hormones are always present $[25,36]$ and constitutive production has been experimentally demonstrated in animals [2, 11]. We note that equations (2.4) and (2.5) imply that continuous stimulation of $\mathrm{GnRH}$ will lead to a sustained maximum production of $\mathrm{LH}$ and $\mathrm{FSH}$, which is not the case in reality and we address this in the extended model of Section 3. However, such uninterrupted stimulation of GnRH does not occur under normal, unperturbed circumstances that the basic model is intended to describe.

The pulsatile nature of GnRH production is a critical part in the regulation of FSH and LH manufacture and release. However, the frequency of the pulses occurs relatively rapidly in comparison to the timescales of follicular development. Exploiting the disparity in timescales, we model the overall effect of pulse frequency and amplitude as a quasi-steady "moving average" concentration, defined for example as

$$
G(t)=\frac{1}{2 T_{s}} \int_{-T_{s}}^{T_{s}} G_{\text {pulse }}(t-\hat{t}) d \hat{t}
$$

where $G_{\text {pulse }}(t)$ is the measured $\mathrm{GnRH}$ concentration and $T_{s}$ is a suitable time span to calculate the moving average, e.g. 1 day. This assumption simplifies significantly the model and distinguishes our approach from other modelling studies. We note that in assuming this formulation for $G$, the model will be limited in describing processes that are solely governed either by GnRH pulse-rate or by the amplitude of released GnRH; however, the model as it stands can be used to describe, for example, processes that produce a maximum response at sub-maximal GnRH frequencies by assuming a unimodal response function of $G(t)$. The output of $\mathrm{GnRH}$ is promoted by estrogen $E$ and inhibited by progesterone $P$ and we write

$$
G=G_{\min }+\left(G_{\max }-G_{\min }\right) H^{+}\left(E ; E_{c G}, m_{E G}\right) H^{-}\left(P ; P_{c}, m_{P}\right),
$$


where $G_{\min }$ and $G_{\max }$ are the maximum and minimum levels of $\mathrm{GnRH}$ and so $G \in\left(G_{\min }, G_{\max }\right)$; the minimum level once again reflects constitutive output. The physiology of GnRH in human is usually inferred from examining the pulsatile LH secretion. The current understanding of GnRH physiology comes from various studies of animal models by experimentally manipulating the hypothalamus and pituitary interactions, which permit validation on $\mathrm{GnRH}$ secretion and the subsequent LH release $[7,17]$. In the absence of available data, we will normalized the concentration $G$ such that $G_{\max }=1$ in the simulations that follow; the real concentration can be derived once the maximum physiological level of $\mathrm{GnRH}$ is known. Estrogen is produced by the follicle and corpus luteum and progesterone mainly by the corpus luteum. In the absence of data to suggest otherwise, we apply the simplest assumptions to model these hormones. We assume that all active cells of the follicle, and similarly of the corpus luteum, have equal capacity to secrete their respective hormones, which means that the rate of production of each of the hormones will be linearly proportional to the follicular mass. We further assume that the production and loss of the hormones are in a quasi-steady equilibrium, so that their levels can be expressed as linear functions of the active follicular masses, hence

$$
\begin{aligned}
& E=\beta_{E}+\beta_{E F} F+\beta_{E C} C, \\
& P=\beta_{P}+\beta_{P C} C
\end{aligned}
$$

where $\beta_{E}$ and $\beta_{P}$ are the background levels of estrogen and progesterone, respectively.

The model now consists of three coupled, nonlinear differential-delay equations (2.1)-(2.3) for variables $F, R$ and $C$. We are most interested in the long-time solutions, as these will represent the model's description of the menstrual cycle. However, to close this system we need to impose a set of initial conditions for $F, R$ and $C$ at $t=0$ and, due to the delay terms in (2.4) and (2.5), functions that define these variables for $t<0$. As will be demonstrated in the following section there are parameter regimes that result with multiple large time attractors, which depend crucially on the starting conditions.

\section{$2.1 \quad$ Numerical results}

The numerical solutions of the differential equations (2.1) - (2.3) are performed using Numerical Algorithms Group (NAG) routine D02EJF, which uses a backward difference formula to integrate a stiff system of first order differential equations over an interval with suitable initial conditions. The delay terms are handled by storing a history table of previous solutions and using interpolation formula. We note that all simulations presented in this paper are started at some time point $t=t_{0}<0$, so that by $t=0$ the long time periodic solutions have fully developed.

The simulations are generated using the parameter values listed in Table 2 in Appendix A; the parameter values being chosen so that simulations produce results matching approximately the 31 day period of the experimental data [25] and agree with most key characteristics of the hormone profiles, namely, the amplitudes of E2, P4, LH and FSH, and the post-ovulatory peak of E2 concentration. To our knowledge, there are no experimental data that can inform any of the model parameters directly. Though it is uncertain to what extent the data values listed in Table 2 reflect biological reality, particularly as many complex processes have been reduced in the modelling assumptions to relatively simple kinetic terms, many of the parameters do have direct biological meaning (e.g. the minimum and maximum levels of FSH and LH) and the values in the table can thus form the basis for comparison when suitable experimental data are forthcoming. 

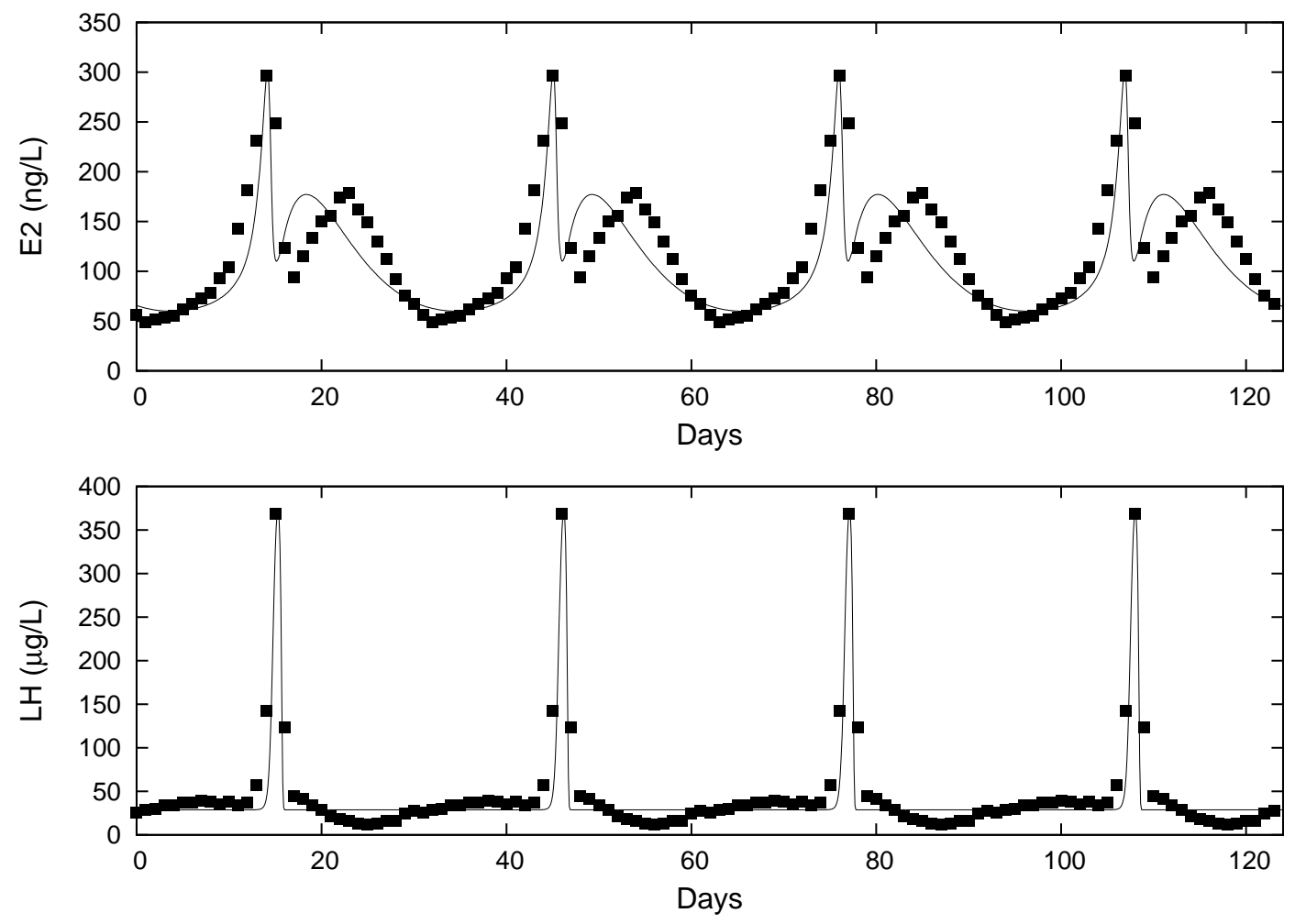

Figure 2: Comparison with data from McLachlan et al. [25] for E2 and LH. The solid curves are simulated solutions of system (2.1) - (2.8) with the parameter values taken from Table 2 (Set 1 ); the initial and past values used are $F(t)=100, R(t)=25$ and $C(t)=25$ for $t \leq t_{0}<0$, and the results shown here are the stable periodic solutions that have evolved in "large time".

A comparison of the simulated E2 and LH profiles with data values extracted from [25] are shown in Figure 2. We observe from this figure that the model predicts the data profiles qualitatively well over the whole of the cycle, however, in quantitative terms, there are parts of the profiles that are not so well matched. The main quantitative difference occurs with the timing of the post-ovulatory peaks of E2 (and P4, not shown) and the variation in the fluctuations of basal concentration level of LH (and FSH, not shown). The post-ovulatory peak is predicted to occur 4-5 days earlier in the current model, this would suggest that there is some form of lag between initial luteal development and E2 (and P4) production, which is not accounted for in the current model. Although we have not found definitive support from the experimental literature, it seems that there are as yet unclassified, distinct stages of development present within each follicular state; the inclusion of which in the current model would produce results closer to the data, at a cost of a more complicated model. This approach was adopted in [4], in which a total of 9 follicular states was considered to generate hormone profiles that agree well with data.

The time dependent profiles of the periodic solutions of all the variables are shown in Figure $3(\mathrm{a})-(\mathrm{d})$. In fact, there are (at least) two large time attracting limit cycle solutions for this set of parameters, the one shown in Figure 3 (and in Figure 2) is what we term the "normal cycle", which has a period of about 31 days with the ovulation event shown here to occur at about day 15. Figure 3(c) shows the concentration of GnRH rising sharply immediately before LH 
(a)

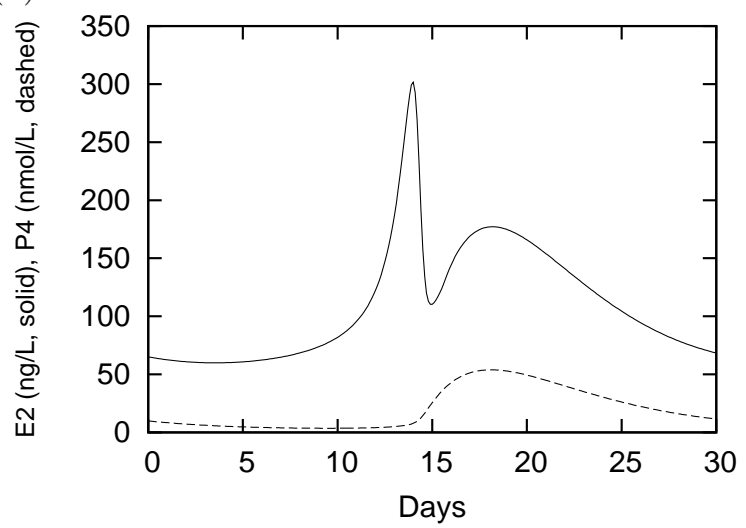

(c)

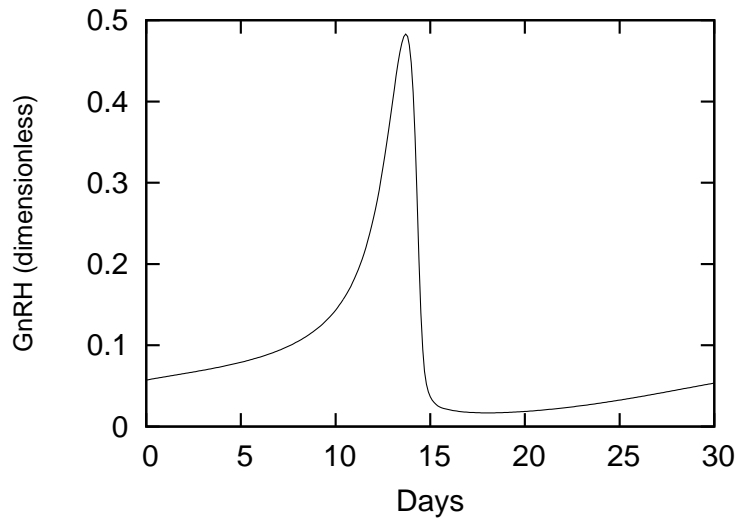

(b)

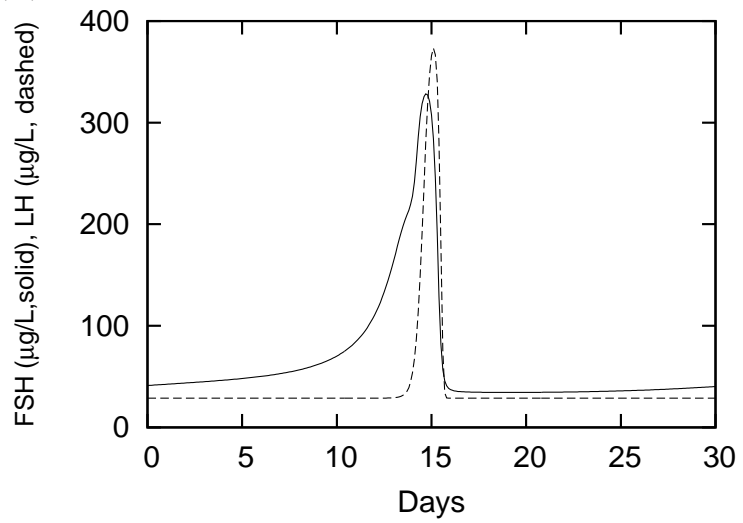

(d)

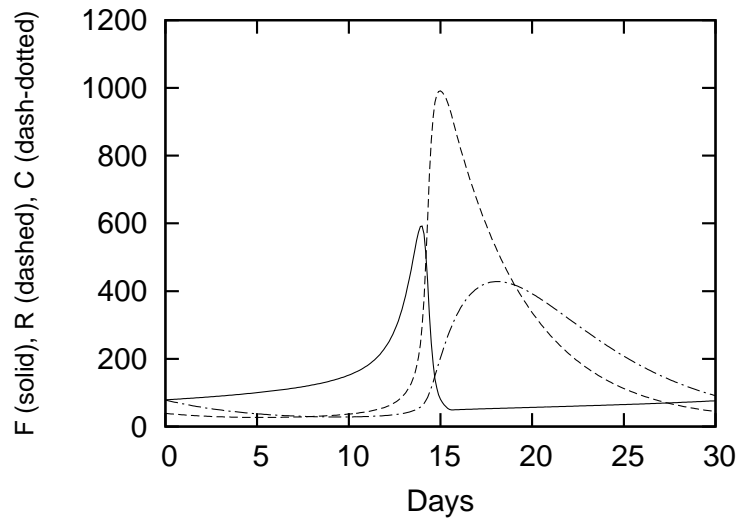

Figure 3: Numerical simulations of the system (2.1) - (2.8) using parameter values and initial/past conditions described in the caption of Figure 2. Note the values of $F, R$ and $C$ are in arbitrary mass units.

surge. It is believed that GnRH pulse rate and amplitude increase near the end of the follicular phase building up to a surge, a phenomenon modelled by Clément and Francoise [6] using a pair of coupled systems of FitzHugh-Nagumo equations. Although the half-life of GnRH is short (approximately 2-4 minutes), with the increase in pulse frequency and surge in the amplitude, a rise in the portal blood concentration of GnRH can be expected prior to the LH surge as represented in Figure 3(c). The expected rapid growth of follicles prior to ovulation is shown in Figure 3(d) together with the profiles of the other ovarian state variables. The corpus luteum mass is predicted here to be maximal about 3 days after ovulation and decays away exponentially to negligible levels after about 10 days.

As stated earlier, there are at least two large-time stable periodic attractors, each dependent on the conditions imposed at $t=0$ and $t<0$. The stability of these attractors were investigated using a combination of Poincaré map (results not shown) and Floquet analysis (discussed in Section 2.2). Figure 4 compares the results of $\mathrm{E} 2$ and $\mathrm{LH}$ for the two periodic solutions resulting from the current model, the solid line being that of the normal cycle discussed above and the dashed curve a second cycle, which we will refer to henceforth as the "abnormal" cycle. We note that the follicular and hormone profiles of the abnormal cycle solutions are not intended, or expected, to reflect biological or clinical reality, as mechanisms beyond those considered 

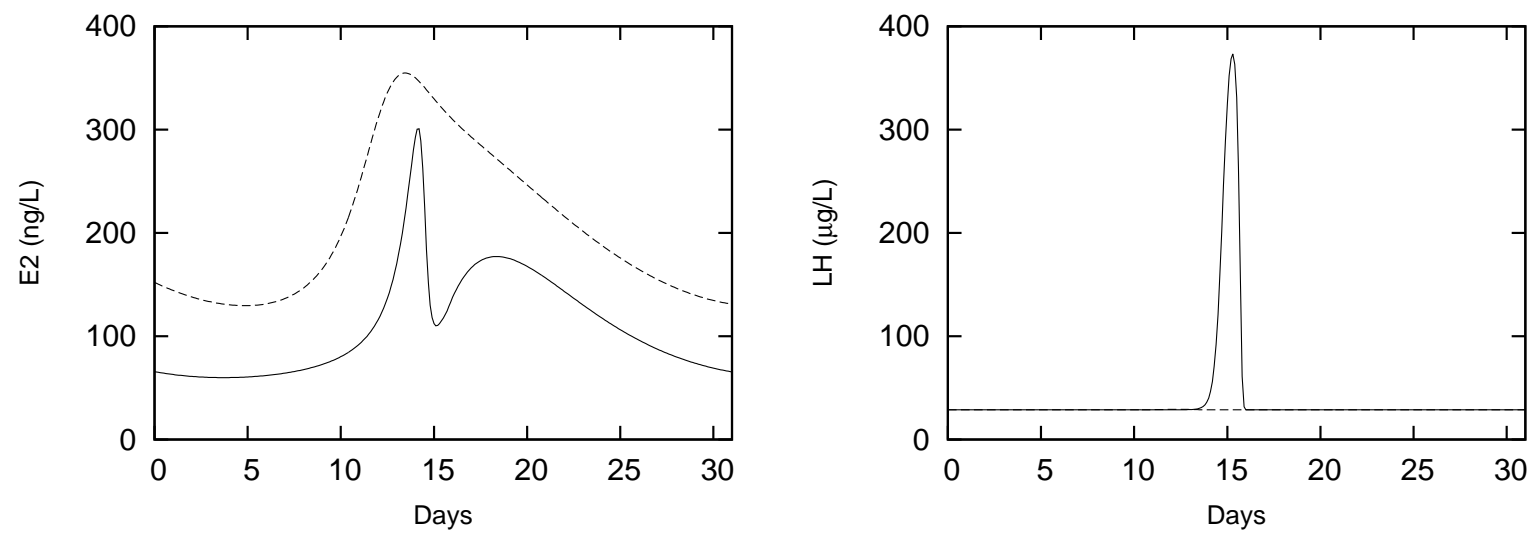

Figure 4: Comparison of the normal (solid line) and abnormal cycles (dashed line) for E2 and LH. Both normal and abnormal cases are simulations of system (2.1) - (2.8) with the parameter values taken from Table 2 (Set 1 ). The results shown here are the large time solutions, using as initial and past values (i.e. $\left.t \leq t_{0}<0\right), F(t)=100, R(t)=25$ and $C(t)=25$ for the normal and $F(t)=100, R(t)=100$ and $C(t)=100$ for the abnormal cycle.

in the current model are likely to be involved. However, it is an interesting feature of the current model that "menstrual irregularities" can be predicted without the need for additional modelling assumptions. The figure shows that over the course of a single period of approximately 27 days, the abnormal cycle lacks the bimodal profile of E2 and the LH surge is barely visible in comparison with the normal cycle. The resulting normal cycle solution can be shifted to the abnormal cycle (or vice versa) by, for example, adding a certain amount of $\mathrm{P} 4$ or E2 continuously for a period of time, reflecting a disruption of hormone activities by treatments or environmental influences; we illustrate this behaviour in Figures 5 and 6. In Figure 5 we show the induction of the abnormal cycle by adding $80 \mathrm{ng} \mathrm{L^{-1 }}$ of E2 continuously for 20 days (starting from day 60 ), where by day 120 the abnormal cycle is clearly apparent. The process is reversed in Figure 6 , where the normal cycle is shown to be restored around day 120 , after $80 \mathrm{ng} \mathrm{L}^{-1}$ of $\mathrm{P} 4$ was administered for 20 days from day 60 . There appears to be little comprehensive data in the literature regarding irregular cycles (e.g. time course hormone measurements) and it is not clear whether or not the "abnormal cycle" predicted in the current model closely reflects reality. Abnormal menstrual cycles, however, are often identified with irregular ovulation which may be attributed to the absence of the LH surge, a characteristic present in the "abnormal cycle" of the current model. We would also like to stress that the abnormal cycle simulated here is induced by the application of exogenous hormones to the system and not a result of physiological disfunction as, for example, in the case of women with PCOS (polycystic ovary syndrome). The study by Marshall et al. [23] suggested that the impaired feedback of progesterone on GnRH leads to rapid GnRH pulsation and the subsequent increased LH and decreased FSH secretion resulting in anovulation (the absence of ovulation). The direct feedback of progesterone on the pituitary is secondary to that of estrogen in a normal cycling woman, and as we intend to focus the study on the treatment of $\mathrm{GnRH}$, the role of progesterone is ignored here. While our model may require further elements to accurately predict treatment for cases such as PCOS, our study here suggests that such abnormality may not be easily corrected by the application of a short course of hormone treatment. 

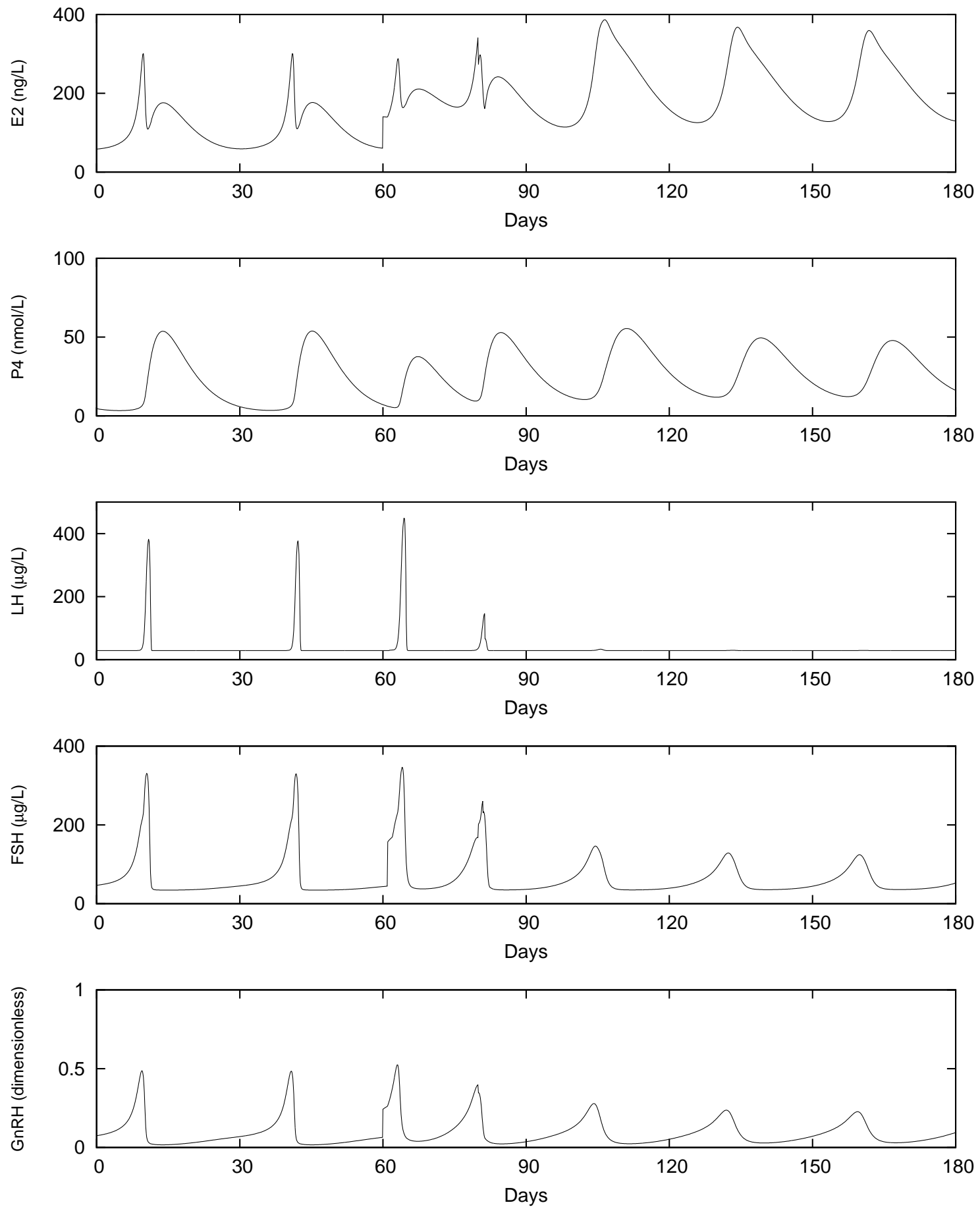

Figure 5: The disruption of a normal cycle by externally applying E2 to the system. The results shown here are the normal cycle solutions that have evolved in "large time" before exogenous E2 is applied, which, for illustration purpose, is set as day 60 in the figure. The parameter values are listed in Table 2 (Set 1 ) and using as initial and past values (i.e. $t \leq t_{0}<0$ ), $F(t)=100, R(t)=25$ and $C(t)=25$. 

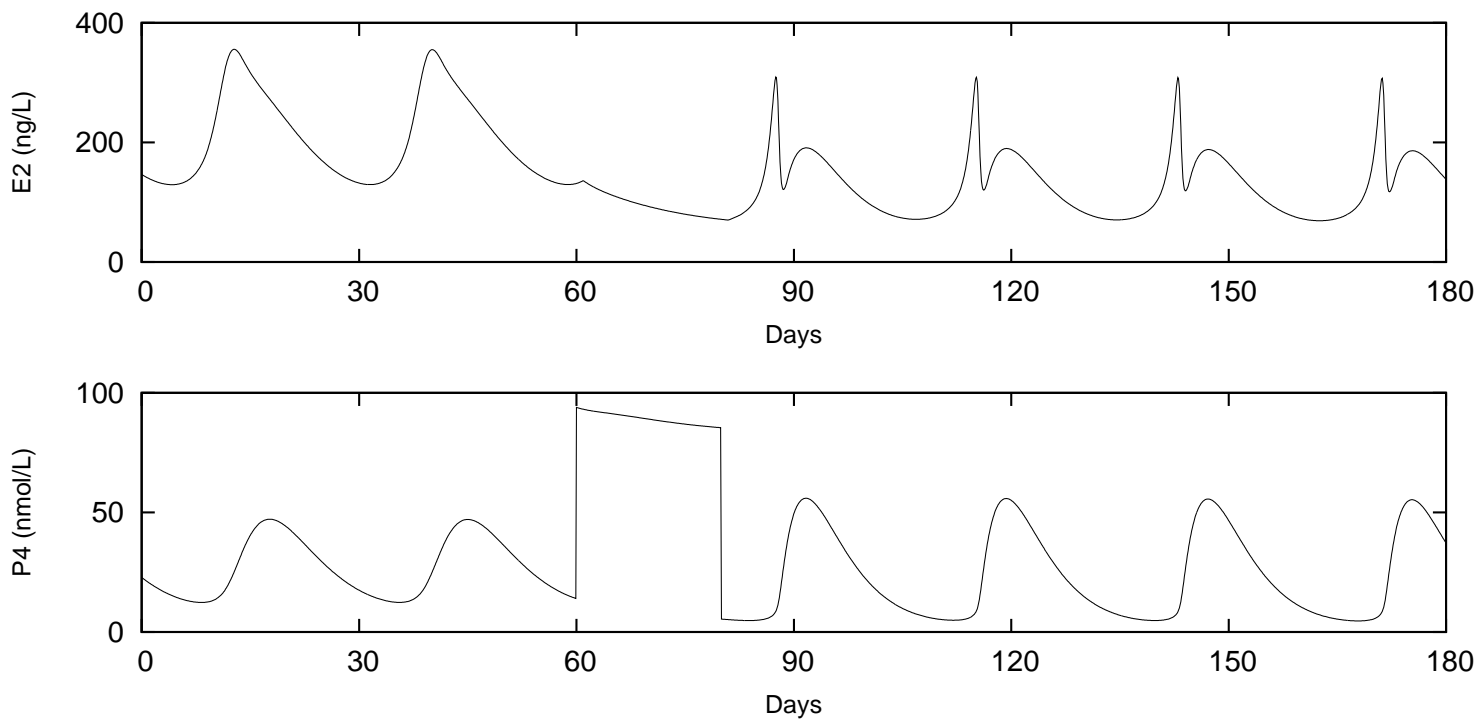

Figure 6: Restoration from the abnormal cycle by externally applying P4 to the system. The results shown here are the abnormal cycle solutions that have evolved in "large time" before exogenous $\mathrm{P} 4$ is applied, which, for illustration purpose, is again set as day 60 in the figure. The parameter values are listed in Table 2 (Set 1) and using as initial and past values (i.e. $\left.t \leq t_{0}<0\right), F(t)=100, R(t)=100$ and $C(t)=100$.

\subsection{Bifurcation analysis}

The existence of multiple large time solutions presented in Section 2.1, prompts us to investigate further the variety of such solutions in parameter space. The discussion here is not intended to be a comprehensive mathematical analysis nor is the choice of the parameters for the illustration below intended to reflect anything of particular biological significance, but aimed at gaining insight into the emergence of such solutions given the complexities arising from the time delay terms. The linear stability analysis of the steady-states of differential-delay equations follow that of ordinary differential equations, though in the former the eigenvalue problem arising from linearisation involves finding the roots of transcendental equations, of which there are an infinite number. To proceed, we make use of a freely available package DDE-BIFTOOL developed by Engelborghs et al. [9, 10], which is a collection of Matlab routines written for carrying out numerical bifurcation analysis, including the application of Floquet analysis, on systems of delay differential equations; the details of the methods used can be found in $[9,10]$ and the references therein. We also adapted our own time-integration program used in Section 2.1 to perform continuation by repeatedly running the time-dependent problem to convergence for each change in parameter; in the case of periodic solutions this is defined to be when for ten successive orbits the period $T_{n}$ satisfies $\left|T_{n}-T_{n-1}\right|<10^{-4}$.

Figures 7 and 8 show a number of aspects of the large-time solutions generated by varying $\beta_{f s}$ (FSH stimulated follicle growth rate constant) and $\beta_{E F}$ (follicular estrogen production constant), with all the other parameters fixed as given in Table 2 . Figure 7 (b) reveals that the distribution of steady-states as a function of $\beta_{f s}$ exhibits hysteresis, with the lower branch consisting of stable steady-states, a middle branch of saddle-points and an upper branch of steady-states which are stable except in the region $0.6183 \lesssim \beta_{f s} \lesssim 3.3062$ bordered by two Hopf bifurcation points. The 
two saddle-node bifurcations are located at approximately $\beta_{f s} \approx 0.5950$ and 1.2043. Figure 7 (a) shows the locations of the real parts of the principal eigenvalues as a function of $\beta_{f s}$ for all the steady-states using DDE-BIFTOOL. Figure 7(c) shows the periods of limit cycle solutions as $\beta_{f s}$ varies. The solid curve shows that of the family of stable limit cycles resulting from the Hopf bifurcations on the upper branch curve in Figure 7(b); the "abnormal cycle" discussed in Section 2.1 belongs to this family. The resulting curve is obtained using DDE-BIFTOOL which performs continuation calculations along the branch of solutions confirming their stability with valid Floquet multipliers. Note that a small section (approximately $0.68 \lesssim \beta_{f s} \lesssim 0.71$ ) of the spike region is dotted in the figure for which DDE-BIFTOOL is not able to confirm the stability of the periodic solutions; as these solutions are not likely to have any biological significance, we will not examine these solutions further and leave this as an open problem. The surface plots of Figure 8, generated by DDE-BIFTOOL, show a sequence of limit cycles in the $F-C$ plane as $\beta_{f s}$ varies between the two Hopf bifurcation points; the plots show no irregularities with the orbits over the region including the area around the spike. In $7(\mathrm{~d})$, the co-ordinates of the Hopf bifurcation points on the upper branch in $\beta_{f s}-\beta_{E F}$ space are illustrated with the periodic solutions existing within the loop. The figure shows that $O(1)$ values of both parameters are required to generate abnormal cycle solutions, indicating that if there is a continued presence of too much or too little estrogen then it is likely that the periodic structure of cycle will be destroyed.

Another interesting aspect of the current study is the second family of stable periodic solutions indicated by the dash-dotted curve in Figure 7 (c), existing in the region $1.43 \lesssim \beta_{f s} \lesssim 2.64$. The "normal cycle" discussed in Section 2.1 exists on this branch. The dash-dotted curve in the figure is generated using our own adapted continuation program described at the beginning of this section and the resulting limit cycle solutions are then imported into DDE-BIFTOOL to evaluate for the Floquet multipliers along this branch; as expected, the largest multiplier is one and the multiplier with the second largest magnitude approaches one as $\beta_{f s}$ approaches the end points of the region. The emergence/annihilation of this second branch of solutions implies the possible presence of fold bifurcations of limit cycles at the end points, suggesting the existence of a corresponding branch of unstable limit cycles; we have not explored this branch of solutions in detail as they are of no interest biologically. However, we note that this "normal" family of limit cycle solutions arises due to the presence of the delay terms and does not exist for sufficiently small values of $\tau_{F S H}$ and $\tau_{L H}$; this is in contrast with the first branch of limit cycle solutions, as they tend smoothly to the limit cycle solutions of the model without time delays (i.e. $\tau_{F S H}=0$ and $\tau_{L H}=0$ ). A key point to observe is that the period (and the variable profiles) change little along the normal cycle branch solutions, indicating a robustness in the solutions to changes in $\beta_{f s}$ over this range; this pattern of robustness remains true also for other parameters.

\section{Modelling GnRH treatment and receptor down-regulation}

The basic model of Section 2 is sufficient in describing hormone interactions under normal physiological conditions while at the same time is capable of simulating hormone disruption of E2/P4 and the necessary treatments for restoring the reproductive system back to its normal function. However, the functional forms used for FSH and LH, given by (2.4) and (2.5), mean that the model will predict sustained output of these hormones to continuous high-levels of GnRH. Consequently, the model will be unable to predict the observed response to the application of GnRH analogs in the treatment of uterine myomas [15] and additional assumptions are needed 
(a)

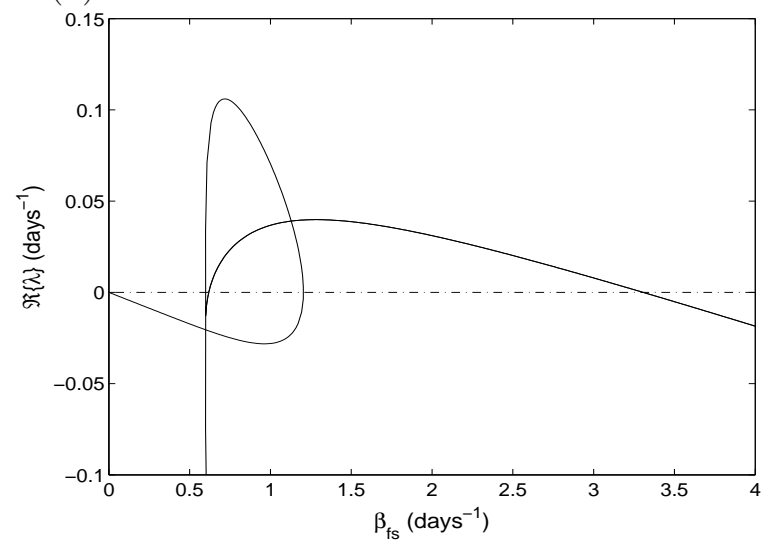

(c)

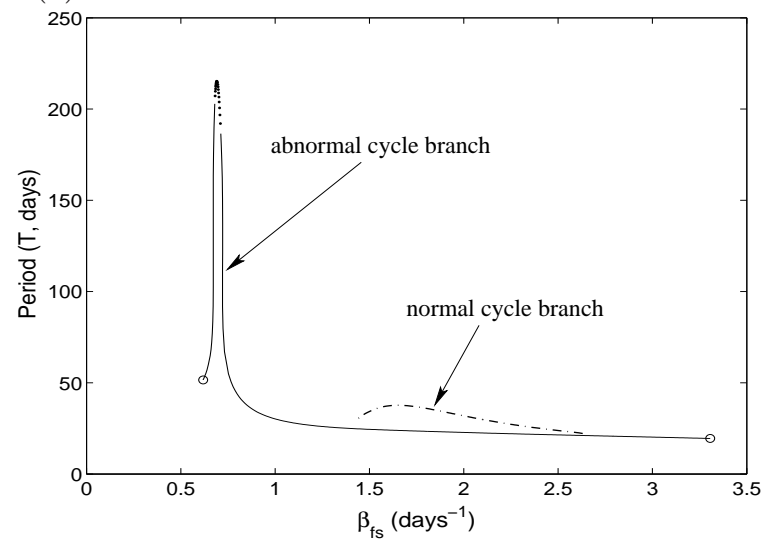

(b)

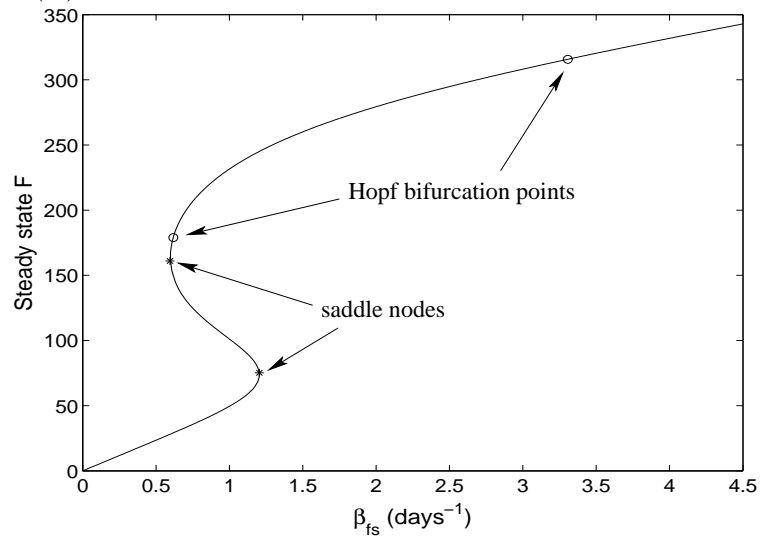

(d)

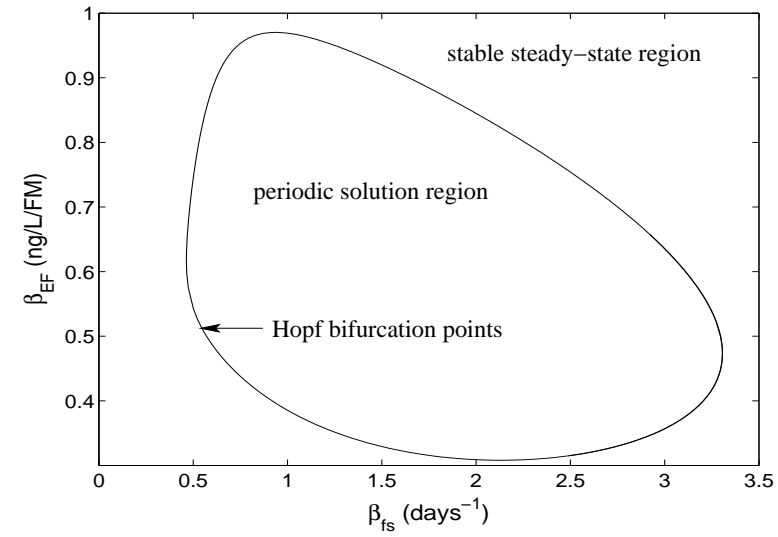

Figure 7: (a) The real part of eigenvalues with $\operatorname{Re}(\lambda)>-1$ as a function of $\beta_{f s}$, derived from linear stability analysis of all steady-states, showing the intersection of the zero axis at 4 places for two Hopf bifurcation points and two saddle nodes. (b) Steady-state solution hysteresis of $F$ showing with two Hopf bifurcation points (o) and two saddle nodes (*). (c) Periods for the two stable limit cycle solution branches containing the abnormal cycle (solid line, circles indicating Hopf bifurcation points) and the normal cycle (dash-dotted line). (d) Co-ordinates of Hopf bifurcation points in $\beta_{f s}$ and $\beta_{E F}$ parameter space. The parameters values used in the simulations are listed in Table 2 (Set 1 ) with $\beta_{f s}$ being the varying parameter for all 4 figures and $\beta_{E F}$ for $(\mathrm{d})$.

to improve the description of events taking place within the pituitary. While pulsatile release of GnRH stimulates the differential production of FSH and LH in the pituitary, continuous stimulation of GnRH results in the desensitisation of the pituitary gonadotrope to GnRH stimulation leading to the eventual suppression of FSH and LH levels. Such response of the pituitary, termed "genuine frequency decoding" [32], is suggested to involve some negative feedback mechanism and various possibilities have been put forward, one, in particular, involves the down-regulation of surface receptors which we will now explore.

GnRH binds with GnRH receptors, a type of G-protein coupled receptor, at the membrane surface of the anterior pituitary cells to activate G-protein and trigger response through a second messenger signalling pathway. The agonists, with higher affinity to GnRH receptors and 

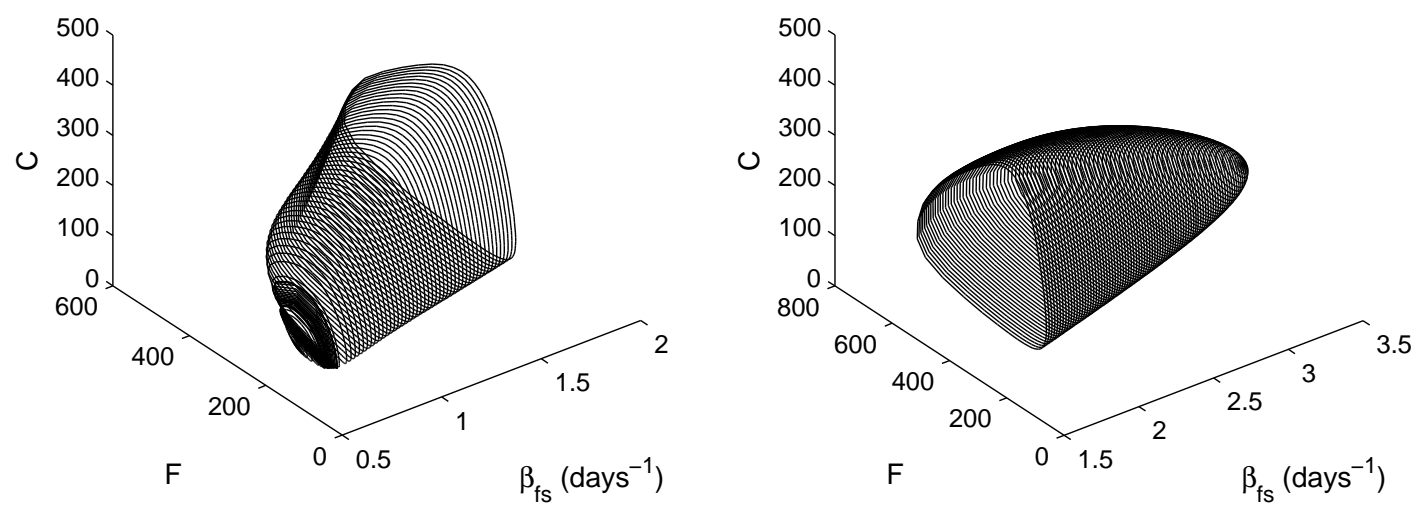

Figure 8: Limit cycle paths in $F-C$ space as a function of $\beta_{f s}$ along the lower branch limit cycle solutions shown in Figure 7. The other parameters values used in the simulations are listed in Table 2 (Set 1).

longer half-life, induce an immediate increase of FSH and LH, followed by E2 and P4 secretion. Continuous stimulation of the agonist together with the feedback of the sex hormones cause the down-regulation of FSH and LH production, preventing the maturation of follicles and the subsequent ovulation, inhibiting the secretion of E2 and P4. The downstream signalling of the receptors is complex and the exact mechanism involved leading to the desensitisation of pituitary response is still very much under debate, however, agonist-induced internalisation and down-regulation of GnRH receptors have been observed [12, 33]. Furthermore, continuous stimulation of $\mathrm{GnRH}$ is reported to reduce MAPK (mitogen-activated protein kinases) activities suppressing the production of FSH and LH and, at the same time, affecting the synthesis of GnRH receptors, possibly paralysing pituitary's response to GnRH stimulation [27, 28]. This idea of receptor down-regulation was taken up in a recent model [35] concerning LH release in response to pulsatile and continuous GnRH stimulation. As a result of constant GnRH exposure, they showed the decay of LH release to zero within hours.

Although the time scale of interest is considerably longer in the current model, we will adopt the similar idea of receptor down-regulation in studying the hormone response to continuous treatment of GnRH agonist; however, for simplicity, we will not explicitly consider the downstream events. We assume that the responsiveness of the pituitary signalling pathway to GnRH stimulation is subject to the $\mathrm{GnRH}$ bound receptor concentration, taken to be proportional to $Q G$, where $Q$ is the membrane receptor density. We modify the model presented in Section 2 by assuming that FSH and LH production is dependent on GnRH bound receptor density, rather than just GnRH level, and write

$$
\begin{aligned}
S= & \left(S_{\text {min }}+\left(S_{\text {max }}-S_{\text {min }}\right) H^{+}\left(G\left(t-\tau_{F S H}\right) Q\left(t-\tau_{F S H}\right) ; G_{c S}, m_{G S}\right)\right) \\
& \times H^{-}\left(E ; E_{c S}, m_{E S}\right), \\
L= & L_{\text {min }}+\left(L_{\text {max }}-L_{\text {min }}\right) H^{+}\left(G\left(t-\tau_{L H}\right) Q\left(t-\tau_{L H}\right) ; G_{c L}, m_{G L}\right) .
\end{aligned}
$$

Receptors, once bound to GnRH, are taken up by the cells to initiate the pathway of reactions leading to the production and release of FSH and LH, thus they are no longer available in the membrane for further binding; we assume this occurs at a rate mutually proportion to $\mathrm{GnRH}$ 
concentration and receptor density. Renewal of the receptors is taken to occur constitutively, being synthesised at a constant rate, and we further assume that there is a natural turn over of unbound receptors. Experimental observation suggests that re-sensitisation of the pituitary to $\mathrm{GnRH}$ following agonist treatments occurs over days or even weeks, which suggests that the renewal of membrane surface receptors occurs on a longer timescale to that of hormone activities and we describe their evolution using a differential equation. The extended model thus consists of four differential equations and we present the system in full, despite the equations for $F, R$ and $C$ being unchanged,

$$
\begin{aligned}
\frac{d F}{d t} & =\left(\beta_{f}+\beta_{f s} H^{+}\left(S ; S_{c}, m_{S}\right)\right) F-\gamma_{f r} F^{2} H^{+}\left(L ; L_{c}, m_{L}\right) \\
\frac{d R}{d t} & =\gamma_{f r} H^{+}\left(L ; L_{c}, m_{L}\right) F^{2}-\gamma_{r c} R \\
\frac{d C}{d t} & =\gamma_{r c} R-\delta_{c} C \\
\frac{d Q}{d t} & =\beta_{Q}(1-Q)-\gamma_{G Q} Q G .
\end{aligned}
$$

We note that $Q$ has been rescaled such that in the absence of $G$, the steady state concentration is given by $Q=1$. As before, equations for $\mathrm{GnRH}$ release rate $G$ and the sex steroids are

$$
\begin{aligned}
G & =G_{\min }+\left(G_{\max }-G_{\min }\right) H^{+}\left(E ; E_{c G}, m_{E G}\right) H^{-}\left(P ; P_{c}, m_{P}\right) \\
E & =\beta_{E}+\beta_{E F} F+\beta_{E C} C \\
P & =\beta_{P}+\beta_{P C} C .
\end{aligned}
$$

For the treatment of myomas, GnRH agonists are often given as monthly dosages. The effects of these agonists are identical to endogenous GnRH, but they have in general a longer half-life. Endogenous GnRH is released directly into the hypophyseal portal blood that links directly to the pituitary whereas exogenous GnRH is injected intramuscularly or subcutaneously and must diffuse from these regions to the blood vessels, before being transported to the pituitary [5]. This process will take time and to assume the immediate availability of agonist following injection could introduce inaccuracies into the model results. A simple model to describe the introduction of the agonist and release into the blood supply is discussed in Appendix B, whereby defining $G_{b}(t)$ to be the agonist concentration in the blood, we obtain

$$
G_{b}(t)=\frac{G_{0} V_{m}}{V_{m}+V_{b}} e^{-\delta_{G} t}\left(1-e^{-k^{*} t}\right)
$$

where $V_{b}$ and $V_{m}$ are the constant blood and intramuscular pool volumes, respectively, $\delta_{G}$ is the agonist degradation rate constant and $k^{*}$ is related to the intramuscular-blood pool transfer rate coefficient. The concentration $G_{b}$ will be similarly normalised, as in Section 3 for Equation (2.6), such that the normalised initial concentration $G_{0}$ is dimensionless and represents the ratio of the initial exogenous GnRH concentration to the maximal endogenous GnRH concentration level. Incorporating this into Equation (3.13) of $G$ gives

$$
\begin{aligned}
G= & G_{\min }+\left(G_{\max }-G_{\min }\right) H^{+}\left(E ; E_{c G}, m_{E G}\right) H^{-}\left(P ; P_{c}, m_{P}\right) \\
& +H\left(t-t_{G}\right) G_{b}\left(t-t_{G}\right),
\end{aligned}
$$

where $H\left(t-t_{G}\right)$ is the Heaviside function, with $t_{G}$ being the time the treatment begins. 


\subsection{Numerical results}

We simulate the treatment of GnRH agonist using the parameter values given by Table 2 (Set 2) taking $V_{b}$ and $V_{m}$ to be equal in Equation (3.16). Note that the extension of the model to include receptor concentration $Q$ requires some minor adjustments in the parameter values to reproduce the normal cycle solutions presented in the earlier sections; see Table 2. Although the shrinkage in myoma sizes is documented during $\mathrm{GnRH}$ treatments, comprehensive data on changes in hormone levels are still lacking. In the absence of available data, the values of the additional parameters are chosen mainly for demonstration purpose showing the effect of 3 monthly dosage treatments of $\mathrm{GnRH}$ on the receptor down-regulation, the subsequent suppression of hormone concentration levels and their recovery, taking into account of the longer half-life of $\mathrm{GnRH}$ agonists $(O$ (hours $)$ ) [7]. Results are again generated using the NAG routine D02EJF and are illustrated in Figures 9. The treatment is applied after the solution has converged to the normal cycle (days 0-30), the result of GnRH concentration in the portal blood is shown in Figure 9 along with the concentration of receptors and other hormones. Note that the endogenous GnRH continues to be generated by the hypothalamus throughout the treatment; its concentration, however, is negligibly small compared to the exogenous $\mathrm{GnRH}$ administered and hence a logged concentration of GnRH is displayed instead.

Three consecutive dosages are administered starting from day 30 (approximately at the beginning of a cycle) with each dosage lasting 31 days. Immediately after the treatment, FSH and LH production is stimulated followed by the secretion of E2 and P4. At the same time, the treatment promotes the degradation of receptors leading to the desensitisation of the pituitary; FSH and LH release is suppressed after the surge at around day 31/32 as a result of receptor down-regulation and, subsequently, E2 secretion is inhibited. Note that although the immediate increase of FSH after the first treatment stimulates the growth of follicles and the subsequent secretion of E2, the concurrent surge of LH quickly converts follicles into scar before they are fully mature. Thus the mass of follicles and that of corpus lutea (not shown) both remain small and, as a result, the production of E2 and P4 are both reduced after the first treatment. This is consistent with clinical observations that the shrinkage of myomas occurs after the first dosage treatment. Note that most studies demonstrated that the maximum reduction in size is achieved within the first three months of therapy with minimal additional benefit derived from longer treatment $[5,15]$. The treatment is discontinued after day 122 (with the last dosage being applied at day 92), following which the system begins to recover and by day 210 hormone concentrations are almost back to pre-treated levels. Following the treatment, the simulation predicts that the normal cycle resumes within 6-7 months of the initial dose; this timescale is in line with the observed return of myomas back to their original, pre-treatment size [5, 15]; we note that to achieve this, the value of $\beta_{Q}$ used reflects a slow overall recovery of receptor concentration to the pre-treatment level (1-2 months). Whether or not this is associated with a return to the normal menstrual cycle following agonist treatments is not certain.

To our knowledge, there appears to be no detailed time-course data on hormone recovery from GnRH-agonist treatment that can be used to compare and validate the model; for example, such data would be very useful to compare with the predicted hormone profiles shown in Figure 9. However, the simulated timescale of recovery described above appears to be consistent with clinical observation, and if new data suggest otherwise, then the timescale can be tuned by adjusting the parameters $\beta_{Q}$ and $\gamma_{G C}$ as appropriate. Though the mechanisms for pituitary recovery is still under debate and the timescales are uncertain, the model results suggest that receptor down-regulation is a sufficient mechanism to reproduce observation. 

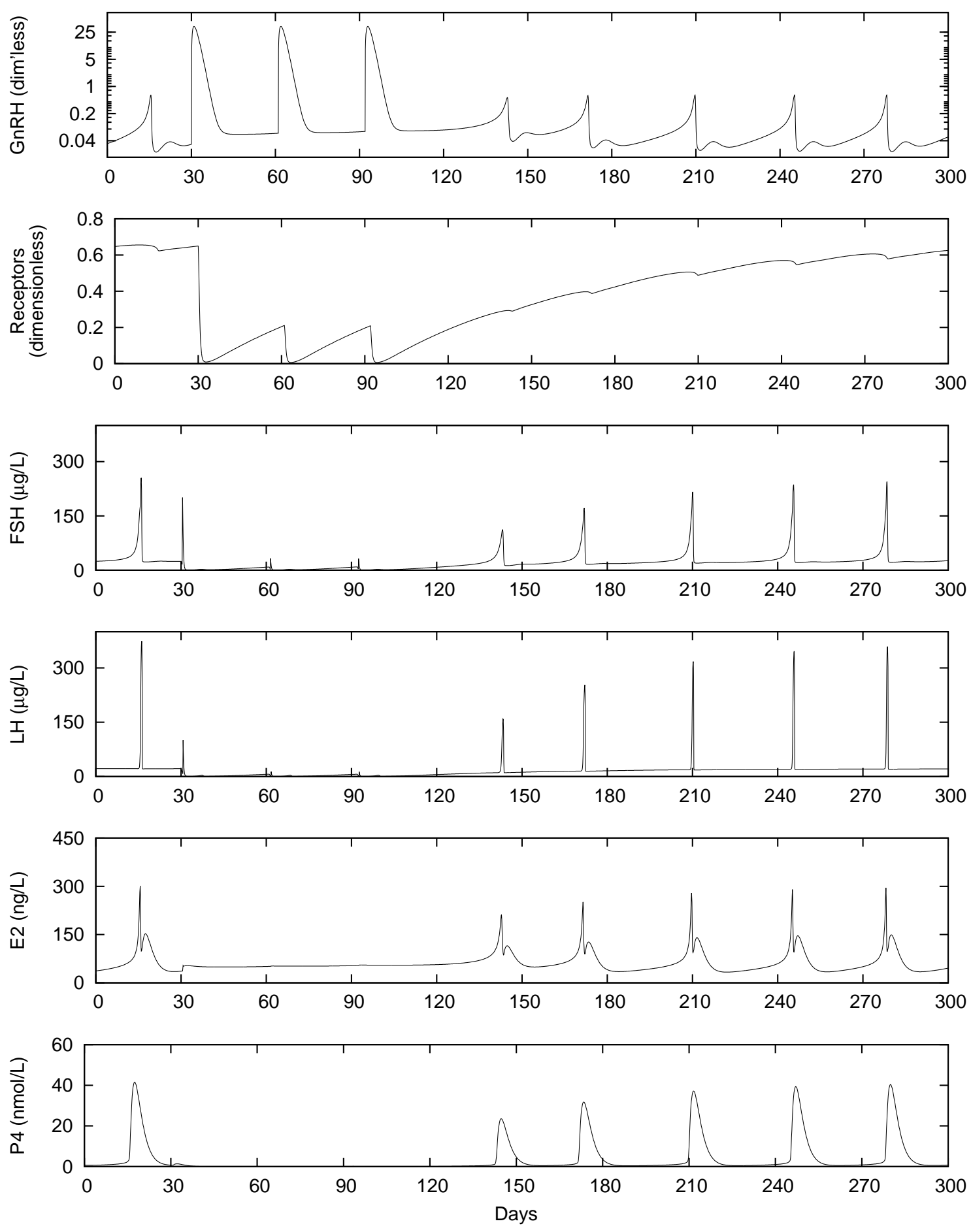

Figure 9: Numerical simulations of the extended model with 3 consecutive monthly dosages of GnRH treatment (note GnRH axis is logged). The parameter values are taken from Table 2 (Set 2), the initial and past values used are $F(t)=100, R(t)=25$ and $C(t)=25$ for $t \leq t_{0}<0$. The results shown here are the normal cycle solutions that have evolved in "large time" before the treatment of exogenous E2 begins, which, for illustration purpose, is set as day 30 in the figure and repeated on day 61 and 92. 


\section{Conclusion}

A mathematical model is proposed to describe the interactions of hormones that are involved in regulating the human menstrual cycle. Chief amongst the aims is to develop a comparatively simple modelling framework, based on biologically relevant mechanisms (using parameters that have a clear biological interpretation), that reproduces, at least qualitatively, the hormone profiles that characterise the cycle. As we intend to extend the model in our future studies, a relatively simple starting model that can be used to investigate perturbations to the cycle is beneficial. The basic model describes the evolution of five dominant hormones and three ovarian stages, which is extended in Section 3 to include $\mathrm{GnRH}$ receptors for the simulation of $\mathrm{GnRH}$ agonist treatment. The simplicity of the model is largely due to the quasi-steady assumptions for the dominant hormones, since their activity works on timescales of hours, whilst the ovarian stages occur over days and weeks. The first stage consists of the three nonlinear delay differential equations, which exhibit multiple large time solutions. The example discussed in detail in Section 2.1 exhibits two asymptotically stable periodic solutions with one (normal cycle) demonstrating the key features of the menstrual cycle and another (abnormal cycle) suggesting the possible abnormality experienced by some women of reproductive age. The resulting numerical simulations of hormone profiles are comparable to the experimental data. Although better agreement can possibly be achieved if more state variables of the ovarian stage are assumed, this will inevitably increase the complexity of an already complicated system. The relative simplicity of the current model enables a more detailed analysis of the solution structures in large time while at the same time is capable of reproducing the myriad of the solutions exhibited, or at least presented, by the more complex model of, for example, [4] consisting of 13 differential equations. The simulations presented in Section 2.1, in which the hormone treatment causes a shift from one cycle to the other, demonstrate the potential of the current model to be used as a basis for investigation into the development and treatment of menstrual irregularities.

The extension to the basic model in Section 3 to incorporate GnRH receptors enabled an investigation into the effect of pituitary desensitisation on the whole menstrual cycle; to our knowledge, no such study has been undertaken before using mathematical modelling. Our model assumes that the dominant process in GnRH agonist induced pituitary desensitisation is $\mathrm{GnRH}$ receptor down-regulation; though, the relative importance of complex downstream events involved in the GnRH receptor signalling is still under debate. Nevertheless, assuming that receptor recovery is slow, the simulated results appear to be consistent with clinical observations, for example, the resumption of the normal cycle within 3-4 months of completion of the treatment. The modelling suggests that the slow recovery of receptor numbers is a sufficient mechanism for densensitisation, though, we stress, this does not preclude the possible involvement of other mechanisms. However, the analysis highlights the potential of using simple mathematical models to investigate hypotheses on the key processes governing the menstrual cycle, enabling new insights to be drawn which may not be achievable with current experimental techniques.

It is important to note that a number of factors believed to take part in regulating the menstrual cycle have not been explicitly considered in the models presented in this paper. Nevertheless, the modelling results reproduce many of the important features and characteristics of the cycle. As discussed earlier, data on hormonal levels during the cycle are generally qualitative and the details of the hormone profiles during the cycle can vary from report to report; the model results presented here are very much consistent with this data. In order to develop these models further, more detailed quantitative data of hormone levels, for example, is required so 
that direct comparisons can be made with the results produced by the model; this will enables us to move forward in validating and refining the modelling assumptions. Nevertheless, we are sufficiently encouraged by the current results to use these simple models as a basis to continue our work in the modelling of the growth and development of myomas. There are, however, numerous questions needed to be addressed in such a complex physiological system, and we believe that the mathematical modelling approach can make significant headway in the biological understanding of this, and related, systems.

Acknowledgement: Both authors would like to thank the Royal Society of London for a International Joint Project research grant. The first author would also like to thank NSC of Taiwan for their financial support. 


\section{Appendix A: Table of parameter values}

\begin{tabular}{|c|c|c|c|c|}
\hline P'meter & Interpretation & units & Set 1 & Set 2 \\
\hline $\bar{\beta} \beta_{f}$ & Background follicle growth rate constant & $1 /$ day & 0 & 0 \\
\hline$\beta_{f s}$ & FSH enhanced follicle growth rate constant & $1 /$ day & $2.1^{*}$ & 3.897 \\
\hline$\gamma_{f r}$ & Follicle rupture rate constant & $1 /$ day $/ \mathrm{FM}^{\ddagger}$ & $0.06^{*}$ & 0.111 \\
\hline$\gamma_{r c}$ & Scar-Corpus Luteum conversion rate constant & $1 /$ day & $0.285^{*}$ & 0.529 \\
\hline$\delta_{c}$ & Corpus Luteum decay rate constant & $1 /$ day & $0.345^{*}$ & 0.64 \\
\hline$\overline{S_{c}}$ & FSH hill function coefficient in follicle growth rate & $\mu \mathrm{g} / \mathrm{L}$ & 150 & 150 \\
\hline$m_{S}$ & FSH hill function exponent in follicle growth rate & - & 2 & 2 \\
\hline$L_{c}$ & LH hill function coefficient in follicle rupture rate & $\mu \mathrm{g} / \mathrm{L}$ & 325 & 325 \\
\hline$m_{L}$ & LH hill function exponent in follicle rupture rate & - & 2 & 2 \\
\hline$S_{\min }$ & Minimum FSH level & $\mu \mathrm{g} / \mathrm{L}$ & 28.5 & 28.5 \\
\hline$S_{\max }$ & Maximum FSH level & $\mu \mathrm{g} / \mathrm{L}$ & 500 & 500 \\
\hline$G_{c S}$ & GnRH hill function coefficient in FSH release & - & 0.453 & 0.453 \\
\hline$m_{G S}$ & GnRH hill function exponent in FSH release & - & 2 & 2 \\
\hline$E_{c S}$ & $\mathrm{E}_{2}$ hill function coefficient in FSH inhibition & $\mathrm{ng} / \mathrm{L}$ & 432 & 432 \\
\hline$m_{E S}$ & $\mathrm{E}_{2}$ hill function exponent in FSH inhibition & - & 4 & 4 \\
\hline$L_{\min }$ & Minimum LH level & $\mu \mathrm{g} / \mathrm{L}$ & 36.4 & 36.4 \\
\hline$L_{\max }$ & Maximum LH level & $\mu \mathrm{g} / \mathrm{L}$ & 676 & 676 \\
\hline$G_{c L}$ & GnRH hill function coefficient in LH release & - & 0.447 & 0.447 \\
\hline$m_{G L}$ & GnRH hill function exponent in LH release & - & 10 & 10 \\
\hline$G_{\min }$ & Normalized minimum GnRH level & - & 0 & 0 \\
\hline$G_{\max }$ & Normalized maximum GnRH level & - & 1 & 1 \\
\hline$E_{c G}$ & $\mathrm{E}_{2}$ hill function coefficient in $\mathrm{GnRH}$ production & $\mathrm{ng} / \mathrm{L}$ & 185 & 185 \\
\hline$m_{E G}$ & $\mathrm{E}_{2}$ hill function exponent in $\mathrm{GnRH}$ production & - & 2 & 2 \\
\hline$P_{c}$ & $\mathrm{P}_{4}$ hill function coefficient in $\mathrm{GnRH}$ inhibition & $\mathrm{nmol} / \mathrm{L}$ & 10 & 10 \\
\hline$m_{p}$ & $\mathrm{P}_{4}$ hill function exponent in $\mathrm{GnRH}$ inhibition & - & 2 & 2 \\
\hline$\tau_{F S H}$ & Response delay for FSH output & day & $1.0^{*}$ & 0.3 \\
\hline$\tau_{L H}$ & Response delay for LH output & day & $1.4^{*}$ & 0.5 \\
\hline$\beta_{E}$ & Constitutive production of $\mathrm{E}_{2}$ & $\mathrm{ng} / \mathrm{L}$ & 0.1 & 0.1 \\
\hline$\beta_{E F}$ & Follicular $\mathrm{E}_{2}$ production per follicular mass & $\mathrm{ng} / \mathrm{L} / \mathrm{FM}^{\ddagger}$ & 0.48 & 0.48 \\
\hline$\beta_{E C}$ & Corpus Luteal $\mathrm{E}_{2}$ production per luteal mass & $\mathrm{ng} / \mathrm{L} / \mathrm{FM}^{\ddagger}$ & 0.36 & 0.36 \\
\hline$\beta_{P}$ & Constitutive production of $\mathrm{P}_{4}$ & $\mathrm{nmol} / \mathrm{L}$ & 0 & 0 \\
\hline$\beta_{P C}$ & Corpus Luteal $\mathrm{P}_{4}$ production per luteal mass & $\mathrm{nmol} / \mathrm{L} / \mathrm{FM}^{\ddagger}$ & 0.12 & 0.12 \\
\hline$\beta_{Q}$ & The intrinsic production rate of $Q$ & $1 /$ day & & 0.01 \\
\hline$\gamma_{G Q}$ & GnRH-GnRH receptor reaction rate & $1 /$ day & & 0.08 \\
\hline$G_{0}$ & Normalized initial concentration of $\mathrm{GnRH}$ agonist & - & & 1000 \\
\hline$\delta_{G}$ & Decay rate of $\mathrm{GnRH}$ agonist & 1/day & & 1 \\
\hline$k^{*}$ & Decay and transport rate of GnRH agonist & $1 /$ day & & 0.1 \\
\hline
\end{tabular}

Table 2: Table of model parameters. "Set 1" are used in the simulations of Section 2 and "Set 2 " are used in the extended model in Section 3, the values that are differ between the two sets are indicated with ${ }^{*}$ in the Set 1 column. ${ }^{\ddagger} \mathrm{FM}$ denotes a suitable unit for follicular and luteal mass. 


\section{Appendix B: Exogenous GnRH agonist modelling}

Exogenous GnRH agonists are injected intramuscularly or subcataneously and gradually diffuse into the surrounding blood vessels and transported to the portal blood to interact with GnRH receptors in the pituitary. To estimate the agonist concentration in the portal blood, we assume that the diffusion of $\mathrm{GnRH}$ from the intramuscular pool to the blood vessels is a much slower process compared to blood circulation. For simplicity, we do not differentiate between the agonist in the blood circulation and that in the portal blood and assume two pools of GnRH agonist: the intramuscular and the portal blood pool. Denoting the blood and intramuscular pool agonist concentrations by $G_{b}$ and $G_{m}$, respectively, we assume they evolve according to

$$
\begin{aligned}
V_{m} \frac{d G_{m}}{d t} & =k\left(G_{b}-G_{m}\right)-\delta_{G} V_{m} G_{m} \\
V_{b} \frac{d G_{b}}{d t} & =-k\left(G_{b}-G_{m}\right)-\delta_{G} V_{b} G_{b}
\end{aligned}
$$

where $V_{b}$ and $V_{m}$ are the blood and intramuscular pool volumes, respectively, $\delta_{G}$ is the agonist degradation rate constant and $k$ is the transport rate coefficient between the two pools. The agonist, with initial concentration $G_{0}$, is injected into the intramuscular pool at $t=0$ and thus $G_{b}(0)=0$ and $G_{m}(0)=G_{0}$; we note that the mass of injected agonist is given by $G_{0} V_{m}$. Adding the two equations we obtain $G_{m}+G_{b}=G_{0} e^{-\delta_{G} t}$ and solving them, the agonist blood pool concentration is

$$
G_{b}(t)=\frac{G_{0} V_{m}}{V_{m}+V_{b}} e^{-\delta_{G} t}\left(1-e^{-k^{*} t}\right),
$$

where $k^{*}=k\left(V_{m}+V_{b}\right) / V_{b} V_{m}$ and we have imposed $G_{b}(0)=0$.

\section{References}

[1] Anderson L. Intracellular mechanisms triggering gonadotrophin secretion. Rev. Reprod. (1996) 1, 193-202.

[2] Apfelbaum M.E. Basal and GnRH-Induced Release and Synthesis of LH and FSH from Incubated Pituitary Glands throughout the 4-Day Estrous Cycle of the Rat. Horm. Res. (1981) 15, 109-121.

[3] Bertram R. and Li Y. A mathematical model for the actions of activin, inhibin, and follistatin on pituitary gonadotrophs. Bull. Math. Bio. (2008) 70, 2211-2228.

[4] Clark L.H., Schlosser P.M. and Selgrade J.F. Multiple Stable Periodic Solutions in a Model for Hormonal Control of the Menstrual Cycle. Bull. Math. Bio. (2003) 65, 157-173.

[5] Cheng M., Chao H. and Wang P. Medical treatment for uterine myomas. Taiwan J. Obstet Gynecol. (2008) 47, 18-23.

[6] Clément F. and Francoise J. Mathematical Modeling of the GnRH Pulse and Surge Generator. SIAM J. Appl. Dyn. Syst. (2007) 6, 441-456. 
[7] Conn P.M. and Crowley W.F. Gonadotropin-releasing hormone and its analogs. Annu. Rev. Med. (1994) 45, 391-405.

[8] DeGroot L. and Jameson J.L. (eds.). Endocrinology (5th ed.). Philadelphia: Elsevier Saunders. pp. 2993-3003.

[9] Engelborghs K. , Luzyanina T. and Roose D. Numerical Bifurcation Analysis of Delay Differential Equations Using DDE-BIFTOOL. ACM T. Math Software (2002) 28, 1-21.

[10] Engelborghs K., Lemaire V., Bélair J. and Roose D. Numerical bifurcation analysis of delay differential equations arising from physiological modeling. J. Math. Biol. (2001) 42, 361-385.

[11] Evans J.J. and Janmohamed S. In vitro basal and GnRH-stimulated secretion of gonadotrophins reflects long-lasting modulatory effects, and peripheral levels are not predicted by pituitary responsiveness to GnRH. Euro. J. Endocrin (1999) 141), 512-520.

[12] Finch A.R., Caunt C.J., Amstrong S.P. and McArdle C.A. Agonist-induced internalization and downregulation of gonadotropin-releasing hormone receptors. Am. J. Physiol. Cell Physiol. (2009) 297, 591-600.

[13] Glidewell-Kenney C., Weiss J., Hurley L.A., Levine J.E. and Jameson J.L. Estrogen receptor $\alpha$ signaling pathways differentially regulate gonadotropin subunit gene expression and serum follicle-stimulating hormone in the female mouse. Endocrin. (2008) 149, 4168-4176.

[14] Gougeon A. Dynamics of follicular growth in the human: a model from preliminary results. Human Reprod. (1986), 1, 81-87.

[15] Gutmann J.N. and Corson S.L. GnRH agonist therapy before myomectormy or hysterectomy. J. Minim. Invasive Gynecol. (2005) 12, 529-537.

[16] Kaiser U.B., Jakubowiak A., Steinberger A. and Chin W.W. Differential effects of gonadotropin-releasing hormone $(\mathrm{GnRH})$ pulse frequency on gonadotropin subunit and GnRH receptor messenger ribonucleic acid levels in vitro. Endocrin. (1997) 138, 1224-1231.

[17] Karsch F.J., Bowen J.M., Caraty A., Evans N.P. and Moenter S.M. Gonadotropin-Releasing Hormone Requirements for Ovulation. Bio. Reprod. (1997) 56, 303-309.

[18] Khadra A. and Li Y. A model for the pulsatile secretion of gonadotropin-releasing hormone for synchronized hypothalamic neurons. Biophys. J. (2006) 91, 74-83.

[19] Kile J.P. and Nett T.M. Differential Secretion of Follicle-Stimulating Hormone and Luteinizing Hormone from Ovine Pituitary Cells following Activation of Protein Kinase A, Protein Kinase C, or Increased Intracellular Calcium. Bio. Reprod. (1994) 50, 49-54.

[20] Keener J. and Sneyd J. Mathematical Physiology, Springer, (1998).

[21] Knollman P.E. and Conn P.M. Multiple G proteins compete for binding with the human gonadotropin releasing hormone receptor. Arch. Biochem. Biophys (2008) 477, 92-97.

[22] Li Y. and Khadra A. Robust synchrony and rhythmogenesis in endocrine neurons via autocrine regulations in vitro and in vivo. Bull. Math. Bio. (2008) 70, 2103-2125. 
[23] Marshall J.C., Dalkin A.C., Haisenleder M.L. and Kelch R.P. GnRH pulses - the regulators of human reproduction. Trans. Am. Clin. Climatol. Assoc. (1993) 104, 31-46.

[24] Maruo T., Ohara N., Wang J. and Matsuo H. Sex Steroidal regulation of uterine leiomyoma growth and apoptosis. Human Reprod. Update (2004) 10, 207-220.

[25] McLachlan R.I., Cohen N.L., Dahl K.D., Bremner W.J. and Soules M.R. Serum inhibin levels during the periovulatory interval in normal women: relationships with sex steroid and gonadotrophin levels. Clin. Endocrin. (1990) 32, 39-48.

[26] Messinis I.E. Ovarian feedback, mechanism of action and possible clinical implications. Hum. Reprod. Update (2006) 12, 557-571.

[27] Naor Z. Signaling by G-protein-coupled receptor (GPCR): Studies on the GnRH receptor. Front. Neuroendocrin. (2009) 30, 10-29.

[28] Pawson A.J. and McNeilly A.S. The pituitary effects of GnRH. Anim. Reprod. Sci. (2005) 88, 75-94.

[29] Reinecke I. and Deuflhard P. A complex mathematical model of the human menstrual cycle. J. Theo. Bio. (2007) 247, 303-330.

[30] Scarselli M. and Donaldson J.G. Constitutive internalization of G-protein-coupled receptors and G-protein via clathrin-independent endocytosis. J. Bio. Chem.(2008) 284, 3577-3585.

[31] Smith M.J. and Jennes L. Neural signals that regulate GnRH neurones directly during the oestrous cycle. Reprod. (2001) 122, 1-10.

[32] Tsaneva-Atanasova K., Mina P., Caunt C.J., Armstrong S.P. and McArdle C.A. Decoding GnRH neurohormone pulse frequency by convergent signalling modules. J. R. Soc. Interface (2012) 9, 170-182.

[33] Vrecl M., Anderson L., Hanyaloglu A., McGregor A.M., Groarke A.D., Milligan G., Taylor P.L. and Eidne K.A. Agonist-induced endocytosis and recycling of the gonadotropinreleasing hormone receptor: effect of $\beta$-arrestin on internalization kinetics. Mol. Endo. (1998) 12, 1818-1829.

[34] Wallach E.E. and Vlahos N.F. Uterine myomas : an overview of development, clinical features and management. Obstet. and Gynecol. (2004) 104, 393-406.

[35] Washington T.M., Blum J.J., Read M.C. and Conn P.M. A mathematical model for LH release in response to continuous and pilsatile exposure of gonadotrophs to GnRH. Theor. Biol. Med. Model. (2004) 1, 9.

[36] Welt C.K., McNicholl D.J., Taylor E.A. and Hall J.E. Female Reproductive Aging Is Marked by Decreased Secretion of Dimeric Inhibin. J. Clin. Endcrin. Metab (1999) 84, 105-111.

[37] Welt C., Sidis Y., Keutmann H. and Schneyer A. Activins, inhibins, and follistatins: From endocrinol-ogy to signaling. A paradigm for the new millennium. Exp. Biol. Med. (2002) 227, 724-752. 
[38] Welt C.K., Pagan Y.L., Smith P.C., Rado K.B. and Hall J.E. Control of follicle-stimulating hormone by estradiol and the inhibins: Critical role of estradiol at the hypothalamus during the luteal-follicular transition. J. Clin. Endcrinol. Metab. (2003) 88, 1766-1711. 